Research Article

\title{
The Shrinking of Beijing and the Rising of Xiong'an: Optimize Population Migration in terms of Transport Service
}

\author{
Hongzhi Lin (i) \\ School of Economics and Management, Southeast University, Nanjing 211189, China \\ Correspondence should be addressed to Hongzhi Lin; linhz@seu.edu.cn
}

Received 13 November 2019; Accepted 16 December 2019; Published 6 January 2020

Academic Editor: Ricardo López-Ruiz

Copyright ( 2020 Hongzhi Lin. This is an open access article distributed under the Creative Commons Attribution License, which permits unrestricted use, distribution, and reproduction in any medium, provided the original work is properly cited.

\begin{abstract}
The population of Beijing has already come to its loading capacity. The China central government plans to build an ideal city named Xiong'an nearby Beijing. The city is expected to work as a carrying hub for noncapital functions of Beijing. The central government does not rush to build before a deliberated urban planning is accomplished. For sustainable development, a difficulty faced by urban planners is that the maximum number of people can be migrated from Beijing to Xiong'an with constraint on level of transport service. This paper developed a specialized bilevel programming model where the upper level is to ensure a predetermined transport service level regarding to population migration, while the lower level is feedback equilibrium between trip generation and traffic assignment. To be more specific, trip is generated by the gravity model, and traffic is assigned by the user equilibrium model. It is well known that the bilevel programming problem is tough and challenging. A try-and-error algorithm is designed for the upper-level model, and a method of successive average (MSA) is developed for the lower-level model. The effectiveness of the model and algorithm is validated by an experimental study using the current transport network between Beijing and Xiong'an. It shows that the methods can be very useful to identify the maximum population migration subject to level of transport service.
\end{abstract}

\section{Introduction}

Beijing is a well-known metropolitan city that is suffering from high housing price, traffic congestion, urban sprawl, air pollution, and water resource shortage. For its sustainable development, the central government of China proposed a millennium strategy to build a state-level new city nearby Beijing in April 1, 2017. The city is located about $100 \mathrm{~km}$ southwest of Beijing. It covers three counties including Xiong, Rongcheng, and Anxin so that it is named as Xiong'an. Its main function is to serve as a development hub for the Beijing-Tianjin-Hebei economic triangle. In addition, noncapital functions of Beijing are also expected to migrate here intensively. However, policy makers usually face a difficulty to determine the amount of migrated population from Beijing to Xiong'an.

There could be several dimensions for sustainable development including economic, social, environmental, and so on. It is worth noting that transport service is a determinant factor for sustainable development. For example, transit-oriented development (TOD) is well accepted in urban planning. It aims to increase public transport and reduce private cars for sustainable urban growth. This paper tries to propose a method to determine the optimal amount of migrated population in terms of private transport services between Beijing and Xiong'an. It is the maximum populations that can be migrated given a level of transport service. The transport system will become unacceptable if more migration is encouraged.

Although the optimal population migration is not investigated yet, the interaction between land use and transport system has been well established where land use is usually indicated by population and employment distribution. Meng et al. [1] proposed a combined land use and transport model to determine the maximum travel demand that can be accommodated by the road network with constraints on the network capacity. It is a bilevel programming model where the upper level problem is the maximization of total travel demand while the lower level problem is a combined trip distribution and traffic 
assignment equilibrium. Lin and Feng [2] developed a bilevel programming model to integrate the land use layout and transport network. The upper level is a sketch layout model which is a nonlinear and multiobjective programming problem. The lower level is a combined trip distribution and traffic assignment model given the land use layout from the upper level problem. The travel times obtained from the lower level problem are fed back to the upper problem. Lee et al. [3] examined the equity issue associated with land use development in terms of travel cost change between different OD pairs. A bilevel programming model was proposed where the upper level problem is travel demand maximization with equity constraints, while the lower level problem is a combined trip distribution and traffic assignment problem. Zhao and Peng [4] explored the interaction between land use allocation and transport system by a bilevel model. The land use allocation strategy generated in the upper level model is fed into the lower transport model. Then, the travel cost and accessibility produced in the lower level model are fed back into the upper level model. Note that the lower level model consists of a logit-type trip distribution and a user equilibrium traffic assignment. Yim et al. [5] formulated a bilevel model where the upper level minimizes the probability of overloading links regarding land use developments and transport network enhancement, while the lower level is a combined trip distribution and traffic assignment model. Qin et al. [6] proposed a bilevel programming model to design a mixed transport network considering sustainable development. The upper level model aims to minimize the total investment costs and vehicle emissions with constraints on land use scale and link load, and the lower level model is the user equilibrium assignment model. Szeto et al. [7] proposed a multiobjective bilevel model to consider social, economic, and environmental dimensions for sustainable road network design. Note that the lower level model is a land-use transport interaction model where the land use is a Lowry-type model, and the transport is a combined modal split and traffic assignment model. Li et al. [8] proposed a feedback procedure for the integrated coevolution of land use and road network. The land use is represented by the distribution of population and employment. Xu et al. [9] proposed an activity-based bilevel programming model to capture the interactions between land use and transport network. The upper level problem is to maximize the total population with a set of given constraints, while the lower level model is a series choice behaviors of commuters. Xiao et al. [10] proposed a bilevel programming model for land use development control where the upper level is the maximization of land use development potentiality subject to an acceptable degree of link saturation, while the lower level is a combined trip distribution and traffic assignment model. Levi et al. [11] formulated a multiobjective bilevel programming model for urban planning where the upper level is monetary cost minimization and travel time minimization with regard to land use layout, while the lower level is a combined trip distribution, mode split, and traffic assignment problem. Zuo et al. [12] developed a bilevel programming model to minimize travel demand by land use planning for compact development. The lower level is a tour-based four-step model to represent the behavior response of travelers to changes from the upper level.

The innovations lie in three aspects. Firstly, the optimal population migration problem has not been explored. The conventional problem is the land use potentiality in terms of a multiplier to an existed travel demand matrix. It is especially meaningful to investigate the population migration between Beijing and Xiong'an. Secondly, a specialized bilevel model is developed where the upper level is to ensure the level of transport service is not worse than a predetermined one with constraints on population migration, while the lower level model is a feedback procedure between trip generation and traffic assignment. Note that trip generation is formulated as the gravity model, and traffic assignment is represented by the user equilibrium model. Thirdly, a tryand-error algorithm is proposed to solve the novel bilevel model. It is easy to understand and use by urban planners in practice.

The structure of the paper is organized as follows. In Section 2, we mainly introduce methodology. It is a bilevel optimization model, in which the upper level is quadratic programming to ensure the level of transport service, and the lower level is a feedback procedure between the gravity model and the user equilibrium model. In Section 3, we introduce the solution algorithm. Section 4 is an experimental study where we simulate the travel between Beijing and Xiong'an in the future and calculate the optimal population immigration considering the level of transport service. Section 5 concludes the paper.

\section{Methodology}

A bilevel optimization model is proposed. The upper level is a quadratic programming where the goal is not worse than a predetermined level of transport service, and the decision variable is the amount of migrated population. The lower level is a feedback procedure between the gravity model and the user equilibrium model. Note that the gravity model is used to predict travel demand between Beijing and Xiong' an, and the user equilibrium model is used to assign the predicted travel demand into the transport network. As travel times are determined endogenously, a feedback procedure is necessary.

2.1. The Upper-Level Model. Before discussing the level of service (LOS) for the transport network, the definitions of LOS for links or sections are listed in Table 1 according to North American highway standards as in the Highway Capacity Manual (HCM). It uses letters A through F, with A being the best and $\mathrm{F}$ being the worst, similar to academic grading. HCM defines LOS of highway sections based on vehicular density, i.e., $v_{a} / c_{a}$, where $v_{a}$ is the traffic volume on link $a$ and $c_{a}$ is the capacity of link $a$.

After the definition of LOS for a basic highway segment, the definition of LOS for the transport network can turn up. It is the weighted sum of all segments by their lengths. Mathematically, it is defined as 
TABLE 1: LOS for a basic highway segment.

\begin{tabular}{lcc}
\hline LOS & Definition & Volume to capacity $\left(v_{a} / c_{a}\right)$ \\
\hline A & Free flow & 0.35 \\
B & Reasonably free flow & 0.55 \\
C & Stable flow & 0.77 \\
D & Approaching unstable flow & 0.92 \\
E & Unstable flow & 1.00 \\
F & Force or breakdown flow & $>1.00$ \\
\hline
\end{tabular}

$$
\operatorname{LOS}=\frac{\sum_{a \in A}\left(v_{a} / c_{a}\right) l_{a}}{\sum_{a \in A} l_{a}},
$$

where $A$ is the set of all links in the transport network, $l_{a}$ is the length of link $a$. The same LOS classification standards as segment are used for the transport network. As the population migrates, the LOS of the transport network should not be worse than a predetermined one.

Therefore, the upper-level model is to determine the maximum amount of population that can be migrated from Beijing to Xiong'an, while assuring a certain level of transport service. Let $\Delta p$ denotes the amount of migrated population. Note that traffic volume $v_{a}$ is an implicit function of $\Delta p$ so that it is expressed as $v_{a}(\Delta p)$. The upperlevel model is formulated as

$$
\begin{array}{ll}
\min & \operatorname{LOS}(\Delta p)=\left(\frac{\sum_{a \in A}\left(v_{a}(\Delta p) / c_{a}\right) l_{a}}{\sum_{a \in A} l_{a}}-\widehat{s}\right)^{2}, \\
\text { s.t. } \quad & p_{b}-\Delta p>\widehat{p}_{b}, \\
& p_{x}+\Delta p<\widehat{p}_{x}, \\
& \Delta p>0
\end{array}
$$

where $\widehat{s}$ is the predetermined level of transport service in terms of volume to capacity, $p_{b}$ is the present population of Beijing, $\widehat{p}_{b}$ is the bottom line of Beijing, $p_{x}$ is the present population of Xiong'an, and $\widehat{p}_{x}$ is the load capacity of Xiong'an. Along with population migration from Beijing to Xiong'an, the transport system performance will deteriorate until the minimum objective. If the population migration continues, the transport system will deteriorate and the objective function will increase. Therefore, it is formulated as a minimization problem, and square is used in case of negative values.

The implicit function $v_{a}(\Delta p)$ is determined by the lowerlevel model. It is a feedback procedure between trip generation and traffic assignment where travel times are determined endogenously.

2.2. The Lower-Level Model. The lower-level model is a feedback procedure between trip generation and traffic assignment. Note that it is the travel demand between Beijing and Xiong'an so that there is no trip distribution issue. In addition, there is also no modal split issue as only private cars are assumed to be used. The feedback loop is shown in Figure 1. The travel demand generated from the gravity model enters into the user equilibrium model. The generated

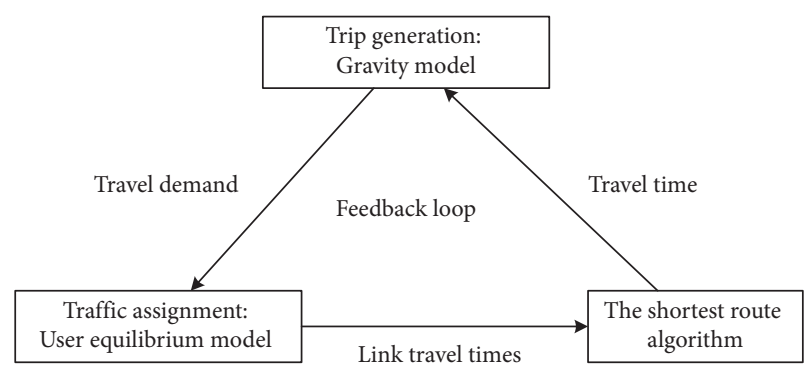

Figure 1: The feedback procedure between nested logit and user equilibrium.

link travel times are then used to calculate OD travel time by the shortest path algorithm. The generated OD travel time enters into the gravity model. The feedback procedure continues until the travel time between Beijing and Xiong'an is determined endogenously.

Although the gravity model is usually used for trip distribution, it is used for trip generation here as there is only travel demand between Beijing and Xiong'an. Gravity model imitates gravitational interaction as described in Newton's law of gravity. It assumes that travel demand between cities is proportional to the product of their populations and inversely proportional to the travel time between them. It can be formulated as

$$
q(\Delta p)=\frac{k\left(p_{b}-\Delta p\right)^{\alpha}\left(p_{x}+\Delta p\right)^{\beta}}{t(\mathbf{v})^{\gamma}}
$$

where $q$ is the travel demand between Beijing and Xiong'an; $p_{b}$ and $p_{x}$ are the present population of Beijing and Xiong'an, respectively; $\Delta p$ is the migrated population consistent with previous definition; $t(\mathbf{v})$ is the travel time between Beijing and Xiong'an which is an implicit function of link travel times $\mathbf{v}$; and $\mathbf{v}$ is a vector form of link travel times $v_{a}$; Note that $v_{a}$ is a function of $\Delta p$ and represented as $v_{a}(\Delta p)$ previously; $k, \alpha, \beta$ are parameters which can be identified empirically.

The generated travel demand is assigned to the transport network by the user equilibrium model. According to Wardrop's first principle of route choice in the field of traffic assignment, it means that traffic arranges itself in congested networks such that all used routes between an OD pair have equal and minimum costs, while all unused routes have greater costs. The user equilibrium can be found by solving an equivalent nonlinear mathematical programming problem. The Frank-Wolfe algorithm is a common way to deal with the problem. The specific formulation is noted as

$$
\begin{array}{ll}
\min & Z(\Delta p)=\sum_{a \in A} \int_{0}^{v_{a}} t_{a}\left(\omega, c_{a}\right) \mathrm{d} \omega, \\
\text { s.t. } \quad \begin{cases}\sum_{k} f_{k}=q(\Delta p), & \forall k, \\
v_{a}=\sum_{k} f_{k} \delta_{a, k}, & \forall a, \\
v_{a} \geq 0, & \forall a, \\
f_{k} \geq 0, & \forall k,\end{cases}
\end{array}
$$


where $A$ is the set of all road links $a$ in the transport network; $v_{a}$ is the traffic volume on link $a ; t_{a}$ is the travel time of link $a$ that is a function of traffic volume $v_{a}$ and road capacity $c_{a}$; and $f_{k}$ is the traffic flow (i.e., number of vehicles) on route $k$ connecting Beijing and Xiong'an; $\delta_{a, k}$ is the link-path incidence relationship which is 1 if link $a$ is on route $k$, and 0 , otherwise. The other notations are consistent with the previous definition. The last two constraints assure no negative traffic.

The produced traffic volumes and travel times in each link from user equilibrium are then used to calculate travel time between Beijing and Xiong'an. It is defined as the shortest travel time among all routes according to Wardrop's first principle of equilibrium. Usually, Dijkstra's algorithm for the shortest path can be adopted here. The generated (shortest) travel time is fed back into the gravity model. The feedback procedure continues until the travel times and traffic volumes in each link do not change anymore. The stable traffic volumes are then used to assess the level of transport service in the upper level.

\section{Solution Algorithm}

The solution of the bilevel optimization model is a wellknown challenge due to difficulties like nonlinearity, nondifferentiability, and nonconvexity. It is a complete NP-hard problem, and the classical algorithms fail. Usually, heuristic methods, though computationally demanding, could be an effective tool to an approximate optimal solution. As only migrated population is the decision variable, it is a line search problem which makes the computation much more easier. The line search approach starts from an initial decision value, finds a descent direction along which the objective function will be reduced, and then computes how far the decision variable should move along that direction.

There are several kinds of line strategies. A kind of heuristic method, try-and-error algorithm, is proposed to solve the bilevel optimization model. The algorithm is specified in detail as follows:

(i) Step 1: identify an initial population migration $\Delta p_{0}$. Note that it is a small value at the very beginning.

(ii) Step 2: generate stable link traffic volumes from the lower-level model. The equilibrium between trip generation and traffic assignment can be found through the method of successive average (MSA).

(iii) Step 3: calculate the objective function $\operatorname{LOS}\left(\Delta p_{0}\right)$ in the upper-level model.

(iv) Step 4: move forward in a step size $h>0$. As a small initial value is used for $\Delta p_{0}$, the moving direction is positive. That is, $\Delta p_{1}=\Delta p_{0}+h$. In order to speed up the moving process, the step size $h$ should not be too small.

(v) Step 5: generate stable link traffic volumes from the lower-level model. The equilibrium between trip generation and traffic assignment can be found through the method of successive average (MSA). (vi) Step 6: calculate the objective function $\operatorname{LOS}\left(\Delta p_{1}\right)$ in the upper-level model.

(vii) Step 7: if $\operatorname{LOS}\left(\Delta p_{1}\right)<\operatorname{LOS}\left(\Delta p_{0}\right)$, it is called try successfully. Then, let $\Delta p_{0}=\Delta p_{1}$ and back to Step 1. If $\operatorname{LOS}\left(\Delta p_{1}\right) \geq \operatorname{LOS}\left(\Delta p_{0}\right)$, it is called error. Then, let $h=h / 4$ to shorten the step size and back to Step 4.

(viii) Step 8: stop rule. If $h<\varepsilon$, stop the iteration procedure. Note that $\varepsilon$ is a predetermined tolerance. If $p_{b}-\Delta p_{1}>\widehat{p}_{b}$ or $p_{x}+\Delta p_{1}<\widehat{p}_{x}$, which means the violation of constraints in the upper-level model, also stop the iteration procedure.

\section{Experimental Study}

The present transport network between Beijing and Xiong'an is shown in Figure 2. The link parameters including free flow travel time, link capacity, and link length are shown in Table 2.

The parameters used for the gravity model in trip generation can be identified empirically. The parameter $k$ is a correction factor which is assumed to be $1 e-7$. The parameters $\alpha$ and $\beta$ are both assumed to be 1 . The parameter $\gamma$ is assumed to be 1.6. According to Beijing Statistical Yearbook 2018, the population of Beijing is $2.17 e+7$ in 2017, i.e., $p_{b}=2.17 e+7$. According to Hebei Economic Yearbook 2018 , the population of Xiong an is $1.10 e+6$ in 2017, i.e., $p_{x}=1.10 e+6$. Therefore, the travel demand in terms of passenger car unit per hour (pch/h) between Beijing and Xiong'an is formulated as

$$
q(\Delta p)=\frac{\left(p_{b}-\Delta p\right)\left(p_{x}+\Delta p\right)}{t(\mathbf{v})^{1.6}} \cdot 10^{-7},
$$

where the initial travel time $t(\mathbf{v})$ is assumed to be 43 minutes, which is the shortest free flow travel time between Beijing and Xiong'an.

The migrated population $\Delta p$ starts from zero and increases at a step size $h=200$ thousand. The migration continues until the objective value in the upper level model fails to decrease. Then, let $h=h / 4$ and continue to move forward. If $h<\varepsilon$, where $\varepsilon$ is assumed to 1000 , stop the movement. The constraints $\widehat{p}_{b}$ and $\hat{p}_{x}$ are both assumed to be $5.00 e+6$. If these constraints are violated, also stop the migration. The bottom line for level of transport service depends on policy makers. They should make a decision on the balance of population migration and transport service. The bottom line $\widehat{s}$ is set to be 1 here which means the traffic flows at the transport network begin to be unstable. If the population migrates further, the traffic flows will become terrible which is disappointing. Policy makers should have bottom line thinking and prevent the breakdown of the transport system.

Travel time and traffic flows are determined endogenously in the lower level model. The travel demand is generated based on the initial travel time and assigned to the transport network by user equilibrium. Then, the new travel time is produced by the shortest path algorithm, i.e., Dijkstra's algorithm. The method of successive average 


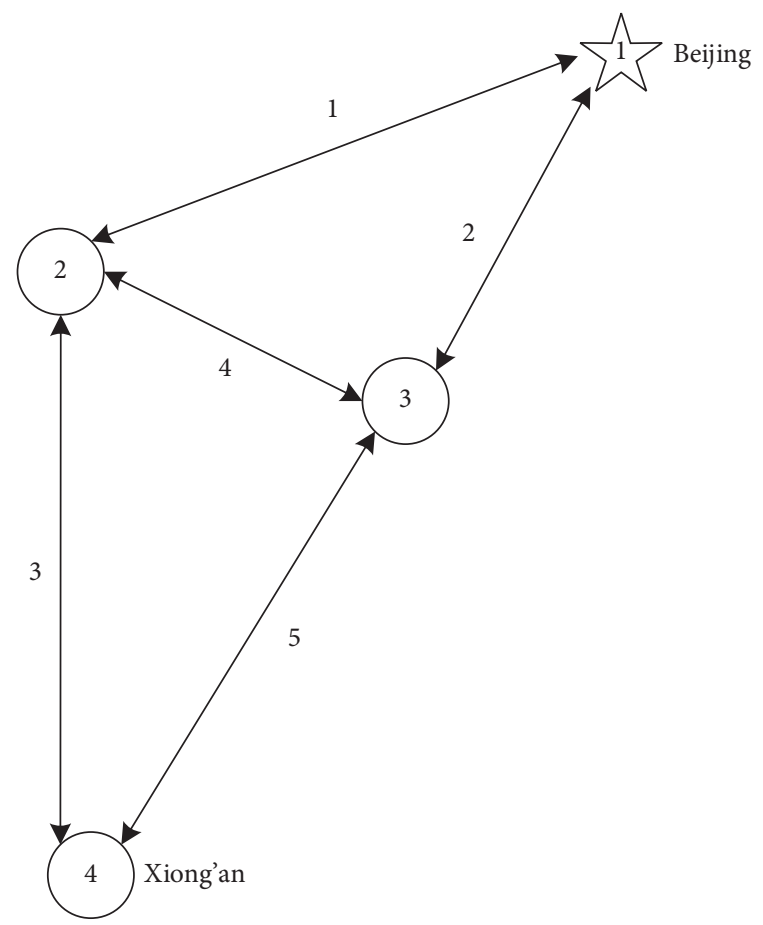

FIGURE 2: The transport network connecting Beijing and Xiong'an.

TABLE 2: Link parameters of the transport network.

\begin{tabular}{lccc}
\hline Link $a$ & $\begin{array}{c}\text { Free flow time } \\
(\mathrm{min})\end{array}$ & $\begin{array}{c}\text { Link capacity } \\
(\mathrm{pcu} / \mathrm{h})\end{array}$ & $\begin{array}{c}\text { Link length } \\
(\mathrm{km})\end{array}$ \\
\hline 1 & 22.5 & 4000 & 45 \\
2 & 22.0 & 4000 & 44 \\
3 & 40.0 & 4000 & 80 \\
4 & 12.5 & 4000 & 25 \\
5 & 21.0 & 4000 & 42 \\
\hline
\end{tabular}

(MSA) on travel times is used where the weights are the reciprocals of the iteration numbers. The iteration procedure continues until the absolute value of the difference between successive travel times is less than 0.01 minute.

To account for congestion effect in the traffic assignment, a link performance function developed by the Bureau of Public Roads (BPR) is adopted. It is formulated as the following:

$$
t_{a}\left(v_{a}\right)=t_{a}^{0}\left[1+\mu\left(\frac{v_{a}}{c_{a}}\right)\right]^{\theta},
$$

where $t_{a}\left(v_{a}\right)$ is the impedance function of a given link $a$ with traffic volume $v_{a} ; c_{a}$ is the road capacity of link $a ; t_{a}^{0}$ is the free flow impedance of link $a$; and $\mu$ and $\theta$ are volume/delay coefficients which can be calibrated empirically. The traditional BPR values for $\mu$ and $\theta$ are 0.15 and 4.0, respectively, which are also used in the simulation study.

The level of transport service with population migration is shown in Figure 3. It demonstrates that the transport network begins to deteriorate as population migration from Beijing to Xiong'an. Given the bottom line of transport service is 1 , the maximum amount of migrated population is

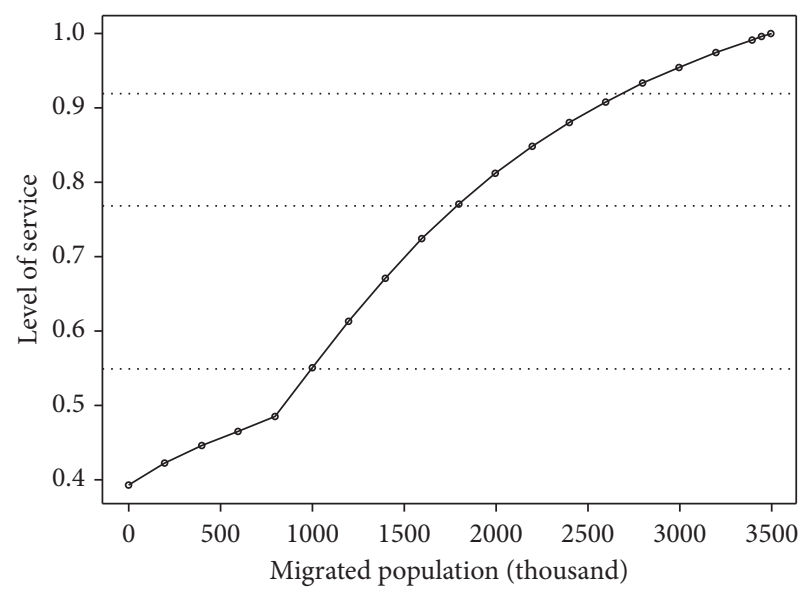

Figure 3: The level of service with population migration.

TABLE 3: The link performances at the maximum population migration.

\begin{tabular}{lcccc}
\hline Link & $\begin{array}{c}\text { Volume } \\
(\mathrm{pcu} / \mathrm{h})\end{array}$ & $\begin{array}{c}\text { Time } \\
(\mathrm{min})\end{array}$ & $\begin{array}{c}\text { Speed } \\
(\mathrm{km} / \mathrm{h})\end{array}$ & $\begin{array}{c}\text { Level of } \\
\text { service }\end{array}$ \\
\hline 1 & 3654 & 24.85 & 108.65 & 0.91 \\
2 & 5670 & 35.32 & 74.74 & 1.42 \\
3 & 3654 & 44.18 & 108.65 & 0.91 \\
4 & 0 & 12.50 & 120.00 & 0.00 \\
5 & 5670 & 33.72 & 74.74 & 1.42 \\
\hline
\end{tabular}

3500 thousand. At this time, the travel time is 69 minutes, and travel demand is $9324 \mathrm{pcu} / \mathrm{h}$. The level of transport service will be unacceptable if more population is encouraged to migrate from Beijing to Xiong'an.

The link performance at the allowed maximum population migration is shown in Table 3. The LOS of link 1 and link 3 is near saturate while that of link 2 and link 5 is already oversaturate. However, the link 4 is not used by drivers that are a waste of resources.

\section{Conclusions}

Nowadays, more and more people live in the metropolitan area, which causes a lot of problems including traffic congestion and air pollution, as well as social problems. Therefore, China's central government plans to reallocate a number of Beijing's noncapital functions to an ideal city named Xiong'an nearby Beijing. The difficulty is how to determine the amount of migrated population for sustainable development. This paper proposed a method to determine the optimal number of immigrates in terms of transport service. It is a novel bilevel optimization model where the upper level is a quadratic programming to assure the level of transport service, and the lower level is a feedback procedure between the gravity model and the user equilibrium model. A try-and-error algorithm is proposed to find the optimal solution.

To demonstrate the effectiveness of the method, an experimental study using the current transport network connecting Beijing and Xiong'an is conducted. The results 
show that it can be a useful tool to help policy makers determine the optimal population migration in terms of transport service. Therefore, it provides both theoretical and practical implications for academics and practitioners.

There are three drawbacks which deserved further efforts. Firstly, the parameters used in the gravity model and the user equilibrium model are not estimated empirically. Although the values are common used in previous research, they could be very different between Beijing and Xiong'an. Secondly, the amount of population migration is based on the current highway network. In fact, the transport network could be expanded and high-speed railway could be introduced. Thirdly, the optimal amount of migrated population should not be determined exclusively by transport service. There are some other dimensions including economic development, social welfare, and environmental protection. Future research can investigate this problem from multiobjective optimization perspective which can provide more valuable implications for policy makers.

\section{Data Availability}

The data used to support the findings of this study are included within the article.

\section{Conflicts of Interest}

The author declares no conflicts of interest.

\section{Acknowledgments}

This research was supported by the National Natural Science Foundation of China (no. 11771078) and the Fundamental Research Funds for the Central Universities (no. 2242019S20012).

\section{References}

[1] Q. Meng, H. Yang, and S.-C. Wong, "A combined land-use and transportation model for work trips," Environment and Planning B: Planning and Design, vol. 27, no. 1, pp. 93-103, 2000.

[2] J.-J. Lin and C.-M. Feng, "A bi-level programming model for the land use-network design problem," The Annals of Regional Science, vol. 37, no. 1, pp. 93-105, 2003.

[3] D.-H. Lee, L. Wu, and Q. Meng, "Equity based land-use and transportation problem," Journal of Advanced Transportation, vol. 40, no. 1, pp. 75-93, 2006.

[4] L. Zhao and Z.-R. Peng, "Integrated bilevel model to explore interaction between land use allocation and transportation," Transportation Research Record: Journal of the Transportation Research Board, vol. 2176, no. 1, pp. 14-25, 2010.

[5] K. K. W. Yim, S. C. Wong, A. Chen, C. K. Wong, and W. H. K. Lam, "A reliability-based land use and transportation optimization model," Transportation Research Part C: Emerging Technologies, vol. 19, no. 2, pp. 351-362, 2011.

[6] J. Qin, L. L. Ni, and F. Shi, "Mixed transportation network design under a sustainable development perspective," The Scientific World Journal, vol. 2013, Article ID 549735, 8 pages, 2013.

[7] W. Y. Szeto, Y. Jiang, D. Z. W. Wang, and A. Sumalee, "A sustainable road network design problem with land use transportation interaction over time," Networks and Spatial Economics, vol. 15, no. 3, pp. 791-822, 2015.

[8] T. Li, J. Wu, H. Sun, and Z. Gao, "Integrated co-evolution model of land use and traffic network design," Networks and Spatial Economics, vol. 16, no. 2, pp. 579-603, 2016.

[9] M. Xu, W. H. K. Lam, Z. Gao, and S. Grant-Muller, "An activity-based approach for optimisation of land use and transportation network development," Transportmetrica B: Transport Dynamics, vol. 4, no. 2, pp. 111-134, 2016.

[10] H. Xiao, J. Gao, and Z. Zou, "Reserve capacity model based on variable demand for land-use development control," Transportation Planning and Technology, vol. 40, no. 2, pp. 199-212, 2017.

[11] Y. Levi, S. Bekhor, and Y. Rosenfeld, "A multi-objective optimization model for urban planning: the case of a very large floating structure," Transportation Research Part C: Emerging Technologies, vol. 98, pp. 85-100, 2019.

[12] T. Zuo, H. Wei, H. Liu, and Y. J. Yang, "Bi-level optimization approach for configuring population and employment distributions with minimized vehicle travel demand," Journal of Transport Geography, vol. 74, pp. 161-172, 2019. 


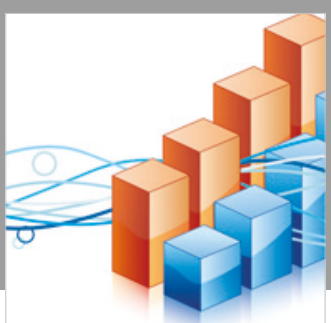

Advances in

Operations Research

\section{-n-m}
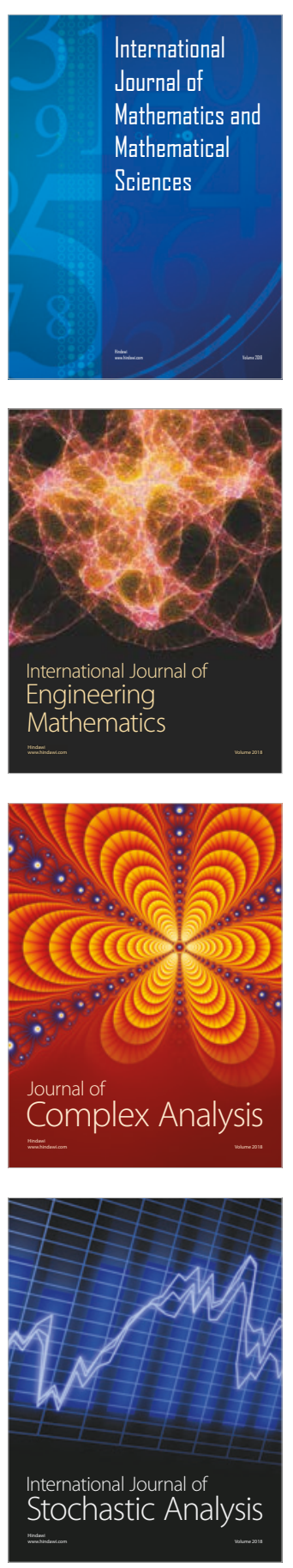
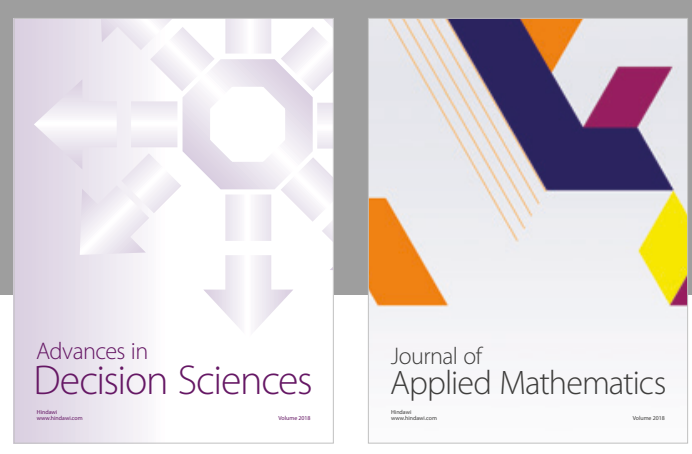

Journal of

Applied Mathematics
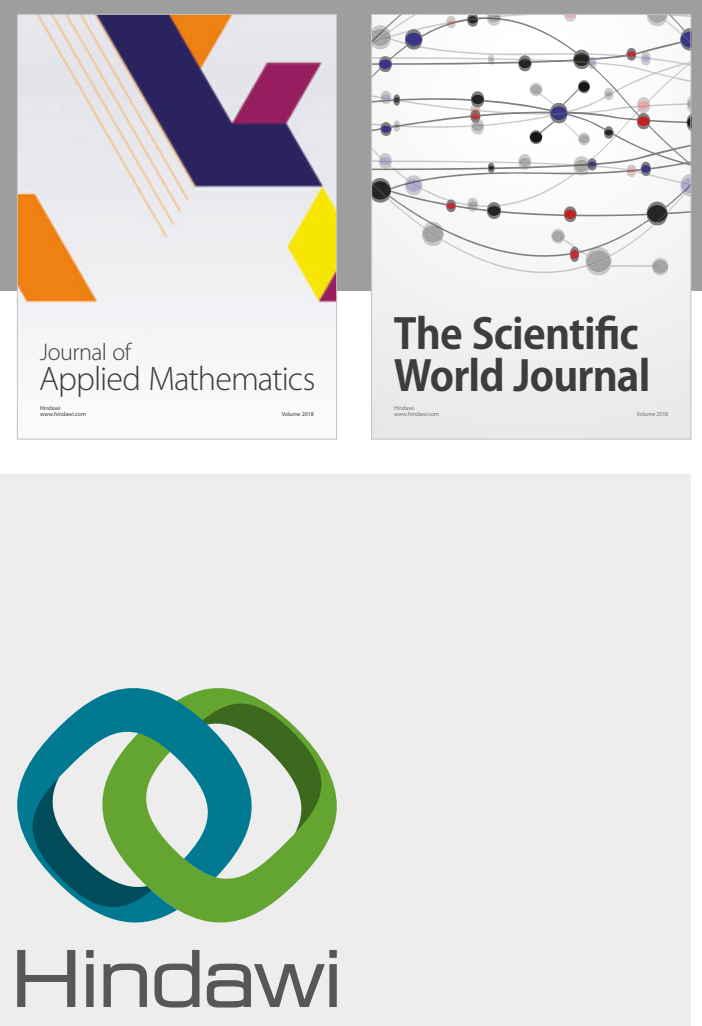

Submit your manuscripts at

www.hindawi.com

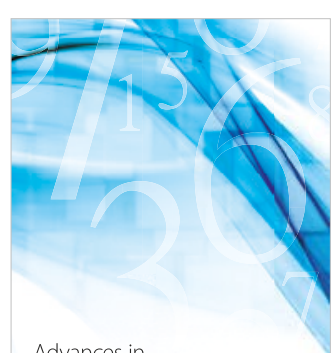

Advances in
Numerical Analysis
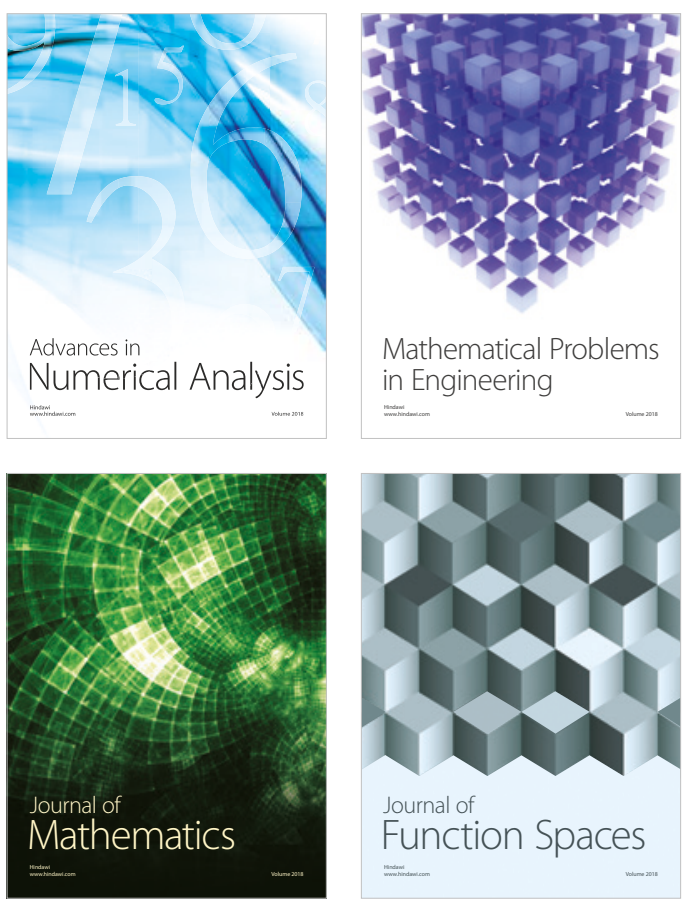

Mathematical Problems in Engineering

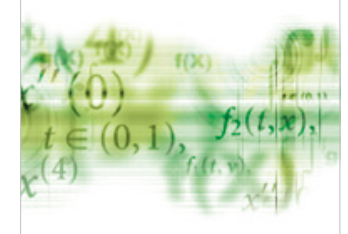

International Journal of

Differential Equations

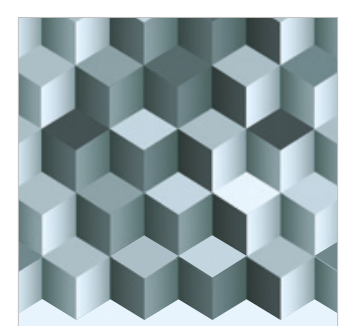

Journal of

Function Spaces

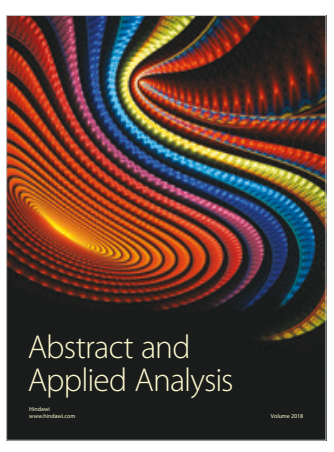

The Scientific

World Journal

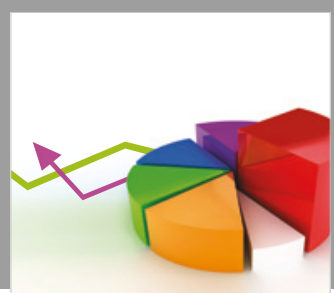

Journal of

Probability and Statistics
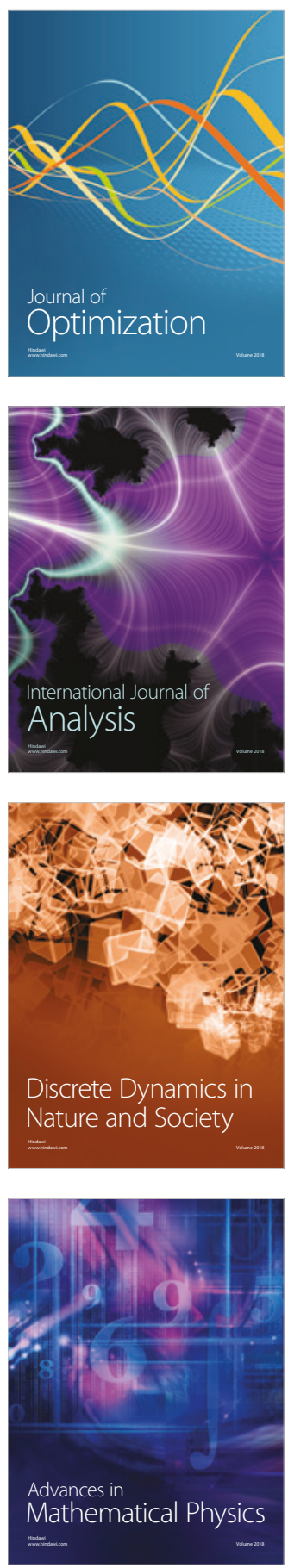